http://dx.doi.org/10.21707/gs.v11.n02a12

\title{
Dinâmica da Comunidade de Oomicetos (OOMYCota) do Riacho Mutum, Demerval Lobão, Piauí, Brasil
}

\author{
Maria do Amparo de Moura Macêdo ${ }^{1 *}$, José de Ribamar de Sousa Rocha ${ }^{2}$
}

\author{
${ }^{1}$ Discente do Programa de Pós-Graduação em Desenvolvimento e Meio Ambiente, PRODEMA, , Universidade Federal do Piaui. \\ ${ }^{2}$ Docente do Departamento de Biologia,Centro de Ciências da Natureza, e do Programa de Pós-Graduação em Desenvolvimento e Meio Ambiente, \\ PRODEMA, Universidade Federal do Piaui, ribamar10@hotmail.com \\ *autor para correspondência: amparo_macedo@hotmail.com
}

Recebido em 02 de março de 2016. Aceito em 16 de abril de 2017. Publicado em 29 de julho de 2017.

REsumo - Os oomicetos são organismos zoospóricos encontrados nos mais diversos ecossistemas aquáticos e terrestres, vivendo geralmente como decompositores da matéria orgânica. Poucos estudos têm analisado a influência de fatores ambientais e a sazonalidade sobre a ocorrência e distribuição desses organismos. Esse estudo analisou a abundância, riqueza, frequência e diversidade dos oomicetos e sua relação com alguns fatores abióticos no riacho Mutum, Demerval Lobão, Piauí, Brasil. De amostras de água e solo coletadas durante o período de estiagem e de chuva em cinco coletas, foram obtidos por meio da técnica de iscagem múltipla 88 isolamentos de oomicetos, agrupados em 20 táxons. O período de estiagem foi o mais propício ao isolamento desses organismos, no entanto o índice de similaridade de Sorensen entre os períodos de estiagem e de chuva mostrou sazonalidade baixa (33\%) e similaridade dos táxons acima da mediana (66,7\%). Todas as espécies isoladas mostraram hábito sapróbio; em substratos celulósicos (80,16\%), queratinosos (9,9\%) e quitinosos $(9,9 \%)$. No solo foi registrado maior ocorrência de oomicetos (63). O aumento da precipitação e a diminuição da temperatura influenciaram de forma positiva a abundância. A comunidade de oomicetos mostrou mudanças em relação a sazonalidade, aos tipos de substratos, compartimentos solo/água, temperatura e precipitação, possivelmente influenciados por especificidade de cada área ou período climático. Esse estudo contribui para ampliar conhecimentos sobre os fatores ambientais que influenciam na dinâmica dos oomicetos.

Palavras-chaves: Fatores Ambientais; Organismos Zoospóricos; Sazonalidade.

\section{Dynamics of Oomycetes community in the Mutum stream, Demerval Lobão, Piaui state, Brazil}

\begin{abstract}
Oomycetes are zoosporic organisms found in various aquatic and terrestrial ecosystems, generally living as decomposers of organic matter. Few studies have examined the influence of environmental factors and seasonality on the occurrence and distribution of these organisms. This study analyzed the abundance, richness, frequency and diversity of oomycetes and their relationship with some abiotic factors in the Mutum stream in the municipality of Demerval Lobão, Piauí State, Brazil. From water and soil samples collected (five collections) during the dry and rainy seasons, 88 oomycete isolations grouped into 20 taxa were obtained by the multiple baiting technique. The dry season was the most conducive to the isolation of these organisms; however, the Sorensen similarity index, between the dry and rainy seasons, showed low seasonality (33\%) and similarity of taxa above the median (66.7\%). All isolated species showed saprobe habit in cellulosic $(80.16 \%)$, keratinous $(9.9 \%)$ and chitinous $(9.9 \%)$ substrates. The soil showed a higher occurrence of oomycetes (63). The increase in rainfall and decreasing in temperature positively influenced the abundance. The oomycetes community showed changes in relation to seasonality, substrates, soil/water compartments, temperature, and rainfall, which were possibly influenced by the specificity of each area or climatic period. This study contributes to further knowledge on the environmental factors that influence the dynamics of oomycetes.
\end{abstract}


Keywords: ENVIRONMENTAL FACTORS; ZoOSPORIC ORgANISMS; SEASONALITY.

\section{Dinámica de la comunidad de Oomicetos en el riachuelo Mutum, Demerval Lobão, Piauí, Brasil}

RESUMEN - Los oomicetos son organismos zoospóricos que se encuentran en diversos ecosistemas acuáticos y terrestres, en general son descomponedores de la materia orgánica. Pocos estudios han investigó la influencia de los factores ambientales y de la estacionalidad sobre la ocurrencia y distribución de estos organismos. Este estudio investigó la abundancia, la riqueza, la frecuencia y la diversidad de los oomicetos y su relación con algunos factores abióticos en el riachuelo Mutum en la ciudad de Demerval Lobão estado de Piauí, Brasil. A partir de las muestras de agua y suelo recogidas durante los periodos de seca y lluvias, en cinco colectas, se obtuvieron mediante la técnica de cebado múltiple, 88 aislamientos de oomicetos, agrupados en 20 grupos taxonómicos. La estación seca fue la más propicia para el aislamiento de estos organismos, sin embargo, el índice de similitud de Sorensen, entre los períodos de sequía y lluvia mostró baja estacionalidad (33\%) y la similitud de los taxones fue superior a la mediana (66,7\%). Todas las especies aisladas mostraron hábito de saprófitos en substratos celulósicos $(80,16 \%)$, de queratina $(9,9 \%)$ y de quitina $(9,9 \%)$. La mayor ocurrencia de oomicetos se registró en el suelo (63). El aumento de la precipitación y la disminución de la temperatura influenciaron positivamente la abundancia. La comunidad de oomicetos mostró cambios en relación con la estacionalidad, los sustratos, los compartimentos del suelo/agua, la temperatura y la precipitación, posiblemente influenciados por la especificidad de cada área o período climático. Este estudio contribuye con la ampliación del conocimiento sobre los factores ambientales que influyen en la dinámica de los oomicetos.

Palabras-clave: Factores Ambientales; Organismos Zoospóricos; Estacionalidad.

\section{INTRODUÇÃO}

Entre os membros de fungos que estão associados com água doce, os organismos zoospóricos compreendem filogeneticamente grupos independentes de táxons pertencentes aos reinos Fungi (exemplo, Blastocladiomycota e Chytridiomycota) e Straminipila (exemplo, Hyphochytridiomycota e Peronosporomycota) e ocorrem principalmente como formas saprotróficas em restos de plantas (Mueller et al. 2004; James et al. 2006; Shearer et al. 2007). São comuns também, além de ambientes aquáticos, nos ambientes terrestres, onde geralmente vivem como decompositores da matéria orgânica (Johnson Jr. et al. 2002; Marano et al. 2011; Jesus et al. 2013; Rocha et al. 2014).

O Filo Oomycota (Reino Straminipila) é representado por organismos zoospóricos, microscópicos, heterotróficos, saprobiontes, mutualistas e/ou parasitas que possuem esporos biflagelados (zoósporos), apresentam celulose ou quitina na composição celular, contém hifas e, além disso, estão presentes nos mais diversos ecossistemas aquáticos e terrestres, e muitas vezes ocupam o mesmo nicho ecológico (Shearer et al. 2007; Kirk et al. 2008; Marano et al. 2011; Nascimento e Pires-Zotarelli 2012; Neuhauser et al. 2012; Jesus et al. 2013; Marano et al. 2014; Beakes et al. 2014).

Czeczuga (2000) afirma que fungos zoospóricos são encontrados em abundância nas águas interiores e tem um papel importante no ciclo de matéria orgânica em várias bacias hidrográficas, com capacidade notória de degradar celulose. Kiziewicz (2004) afirma que os fungos zoospóricos podem ser encontrados em vários tipos de reservatórios de água, colonizando folhas, ramos e galhos de árvores, além de plantas herbáceas e material animal caído dentro da água, contribuindo para a mineralização da matéria orgânica encontrada em vários corpos d'água. 
Pouca informação está disponível sobre os fatores que influenciam a ocorrência e distribuição dos organismos zoospóricos (Sparrow 1968; Dick 1976; Dix e Webster 1995). No entanto, de acordo com Marano et al. (2011) os ecossistemas aquáticos são sujeitos a flutuações em parâmetros ambientais, principalmente devido às alterações sazonais e atividades humanas. Dessa forma, fatores ambientais podem influenciar na distribuição e ocorrência de espécies mudando a dinâmica do ecossistema. Em muitos casos, a sazonalidade das espécies tem sido atribuída à variação dos fatores ambientais (Prabhuji 2011).

Estudo realizado em Las Cañas, Argentina, sugeriram que a maior abundância e diversidade da comunidade de organismos zoospóricos foram obtidos sob baixas temperaturas da água, altas concentrações dos níveis de nutrientes, considerando que a maior riqueza de espécies e frequência estavam relacionadas com temperaturas moderadas e abundância de nutrientes (Marano et al. 2008). Ainda assim, esses mesmos autores enfatizam a importância de mais estudos relacionados à ecologia desses organismos.

No Brasil, como os estudos relacionados aos oomicetos ainda são poucos, quando levamos em conta a grande extensão territorial do país, e vir sofrendo com a intensificação das atividades humanas que ameaçam a biodiversidade, esse estudo faz-se importante, pois representa uma análise ecológica objetivando verificar a dinâmica da comunidade de oomicetos presentes no riacho Mutum, Demerval Lobão Piauí, Brasil.

\section{MATERIAL E MÉTODOS}

O assentamento Mutum está localizado na zona rural do município de Demerval Lobão, Piauí, distante $42 \mathrm{~km} \mathrm{da}$ capital Teresina (Figura 1). O riacho Mutum tem duas nascentes e é afluente do rio Parnaíba. A vegetação do entorno caracteriza-se por uma área de transição entre cerrado e mata de cocais (IBGE 2010).

Figura 1 - Mapa de localização do Assentamento Mutum no município de Demerval Lobão, Piauí.
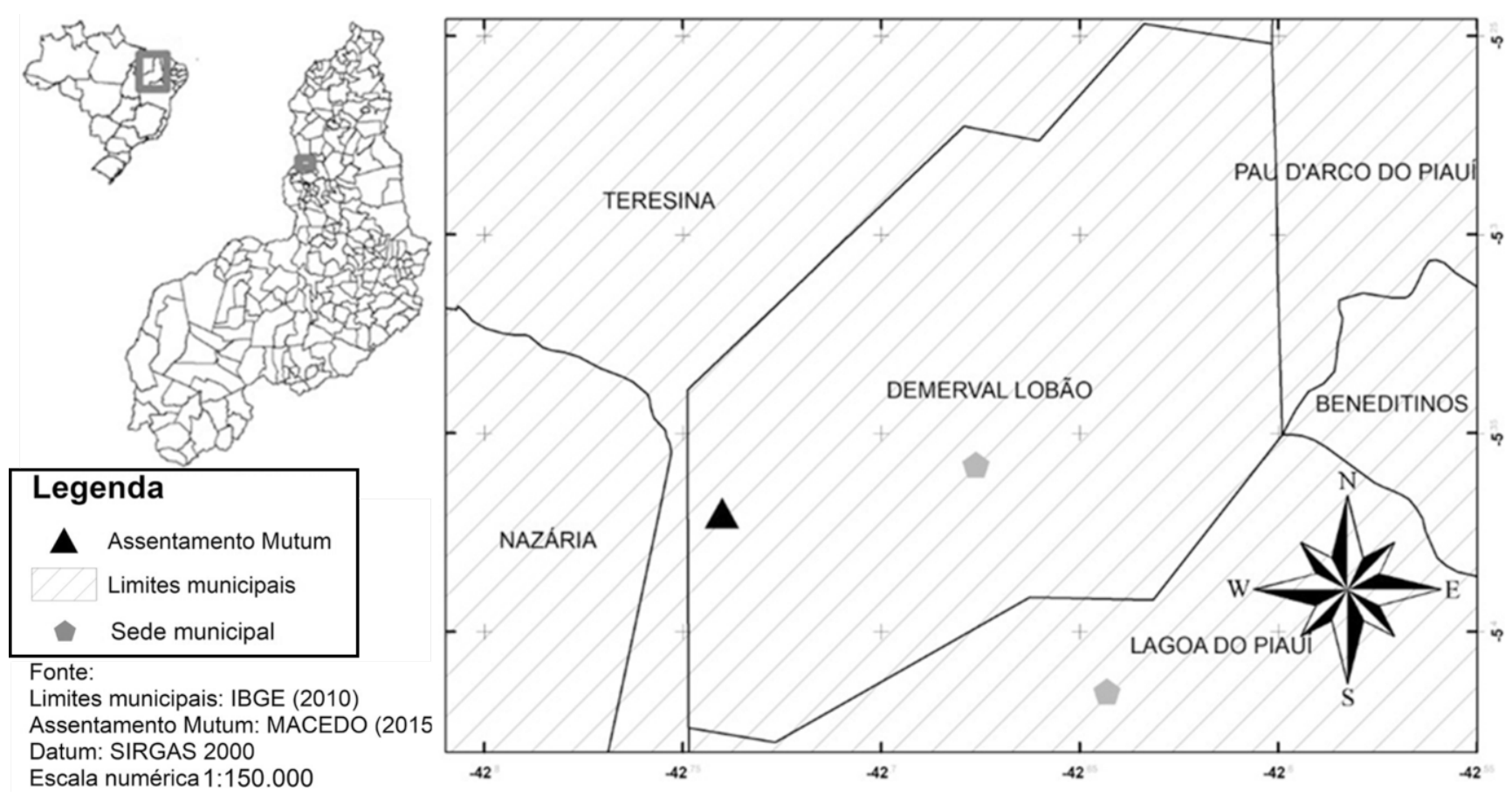
Foram realizadas cinco coletas bimestrais de amostras de água e de solo, em cinco pontos distribuídos às margens do riacho Mutum, escolhidos pela proximidade com o assentamento, nas seguintes coordenadas

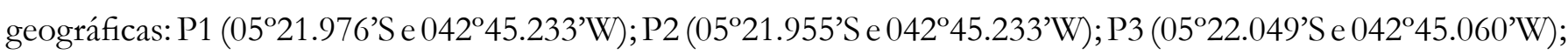

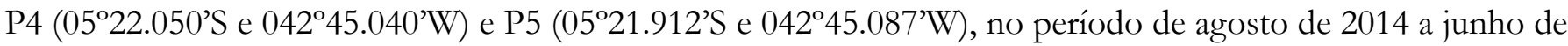
2015.

As amostras foram processadas no laboratório por meio da técnica de iscagem múltipla, para avaliar a composição de espécies, frequência e abundância de oomicetos(Sparrow 1960; Stevens 1974; Milanez 1989). Alíquotas $(30 \mathrm{~mL})$, de cada amostra de água coletada, foram despejadas em placas de Petri e adicionadas iscas celulósicas (semente de sorgo, palha de milho, epiderme de cebola, papel filtro e papel celofane), queratinosas (ecdise de cobra, fio de cabelo e escama de peixe) e quitinosas (asa de cupim). Para a preparação das amostras de solo, $30 \mathrm{~g}$ foram colocadas em placas de Petri com $50 \mathrm{~mL}$ de água destilada esterilizada e também iscada com os mesmos substratos supra citados. Cada placa de Petri foi considerada uma unidade amostral. Estas amostras assim montadas foram incubadas em temperatura ambiente $\left(30-32{ }^{\circ} \mathrm{C}\right)$, e as iscas examinadas ao microscópio a cada sete dias, até completar 42 dias, para a confirmação da presença de oomicetos (ocorrência), que foi cultivado para a identificação taxonômica.

A frequência ( $F$ ) foi determinada para o total de coletas (a coleta consiste de todas as amostras colhidas dos pontos em uma mesma data) e para o total de amostras (a amostra consiste de cada coleta realizada em um ponto), sendo, respectivamente, a frequência de cada ponto de coleta calculada como a percentagem de coletas nas quais uma determinada espécie estava presente ou a percentagem de amostras em que uma dada espécie ocorreu, de acordo com Letcher e Powell (2001).

O índice de Sorensen (Is) foi calculado para análise da similaridade das espécies entre os períodos de chuva e de estiagem. As medidas de similaridade foram calculadas com dados de ocorrência das espécies nas populações, considerando as unidades amostrais de ocorrência (Müller-Dombois e Ellemberg 1974): Is (\%) = 2C / A + B. 100. Onde, $\mathrm{A}=$ número de espécies na estação/compartimento 1; $\mathrm{B}=$ número de espécies na estação/compartimento 2; e $\mathrm{C}=$ número de espécies em comum para ambas as amostras. Se Is $=0 \%$, sem similaridade e Is $=100 \%$, completa similaridade.

As espécies foram agrupadas em cinco níveis de intervalos de frequência, de acordo com a escala de BraunBlanquet: ocorrência ubíqua de 100-80,1 \%; comum de 80-60,1 \%; presentes de 60-40,1 \%; escassa de 40-20,1 \%; e rara de 20-0,1 \% (Kershaw 1973; Letcher e Powell 2001, 2002).

\section{RESUltados E DisCussão}

No estudo da comunidade de oomicetos realizado no riacho Mutum, por meio do método de isolamento desses organismos pela técnica de iscagem múltipla, com emprego de nove tipos diferentes de substratos orgânicos, em cinco pontos da área de estudo, abrangendo cinco coletas, resultou em 225 exames iniciais dos substratos, dos quais, 111 foram positivos para colonização por oomicetos. Estas colonizações representam 49,34\% dos exames, indicando um rendimento mediano do processo de isolamento empregado. Considerando que em outros estudos, utilizando o mesmo método de iscagem, houve maior número de isolamentos, possivelmente, seriam as necessidades nutricionais dos oomicetos dos locais amostrados que poderiam não ter sido totalmente atendidas e ser diferentes das iscas disponibilizadas (Marano et al. 2008; Kiziewicz 2012; Nascimento et al. 2012). 
A Abundância (A) total foi de 88 isolamentos de oomicetos (Tabela 1). O período de estiagem (agosto a dezembro, coletas 1, 2 e 3) mostrou-se mais favorável ao isolamento, contabilizando 46 desses organismos, entretanto, também apresentou a coleta menos abundante, a terceira, com apenas 11 ocorrências. No período de chuvas (março a maio, coletas 4 e 5) foram obtidos 42 isolamentos, dos quais 25 foram da quarta coleta, a mais abundante. Apesar da pouca diferença no número de isolamentos entre os períodos de estiagem e de chuva nesse estudo, já foi observada ocorrência semelhante no Brasil. Nascimento et al. (2011), pesquisando ocorrência e distribuição de organismos zoospóricos no cerrado brasileiro, relatam maior abundância desses organismos no período de estiagem.

A Riqueza (S) foi representada por 20 táxons (Tabela 1), sendo 14 espécies obtidas no período de estiagem e 14 no período de chuvas. Esses dados diferem daqueles relatados por Rocha (2002) e Nascimento (2010), que observaram a maior riqueza no período chuvoso. No entanto, contrariamente, Nascimento et al. (2011) relataram maior riqueza no período de estiagem. Apesar de apresentar micota diversa, poucos táxons foram dominantes (Achlya proliferoides Coker, Pythiogeton ramosum Minden e Pythiogeton dichotomum Tokunaga), assim como também ocorreu em pesquisas realizadas por Nascimento et al. (2011, 2012) e Jerônimo et al. (2015), no Brasil.

A Diversidade (D) de oomicetos observada foi composta por nove gêneros, que se distribuíram em 20 espécies (Tabela 1): Achlya (A. Alagellata Coker, A. proliferoides Coker e A. prolifera C. G. Nees), Aphanomyces (A. helicoides Minden, A. keratinophylus (M. Ôkubo e Kobayasi) R. L. Seym. e T. W. Johnson e Aphanomyces sp), Brevilegnia (B. linearis Coker e B. longicaulis Johnson), Dictyuchus (D. pseudodyction Coker e Braxton e Dictyuchus sp), Globisporangium (G. echinulatum Matthew Stud, G. mamillatum Meurs e G. perplexum Kouyeas e Theoa), Leptolegniella (L. keratinophila Huneycutt), Plectospira (P. myriandra Dreschsler), Pythiogeton (P. dichotomum Tokunaga, P. ramosum Minden, P. uniforme A. Lund e P. utriforme Mind) e Saprolegnia (S. luxurians Bhargava et Srivastava). Achlya proliferoides (20) foi a espécie mais abundante, seguida por Pythiogeton ramosum(13). Ocorrendo em todas as coletas, Pythiogeton ramosum foi também a espécie mais constante. Maranoet al. (2008), em estudo realizado em Las Cañas, Argentina, com organismos zoospóricos, obtiveram diversidade de oomicetos composta por seis gêneros e sete espécies. Já Nascimento e Pires-Zotarelli (2012), em áreas de cerrado na Reserva Biológica de Mogi Guaçu, São Paulo, encontraram diversidade de oomicetos composta por 11 gêneros e 16 espécies, onde Pythiogeton ramosum foi mais frequente e abundante, esses dados tornam-se mais próximos dos obtidos nesse estudo. Jesus et al. (2013) também relatam a ocorrência de oomicetos no Parque Estadual das Fontes do Ipiranga, São Paulo, onde 11 espécies foram identificadas em nível específico. Na literatura especializada, é possível perceber que os relatos de oomicetos são escassos e, portanto, é recomendável a realização de estudos taxonômicos da diversidade desses organismos em todos os biomas representados no país e, principalmente, em regiões que se têm poucos registros, considerando que muitos habitats estão se alterando e conhecer melhor a diversidade desses organismos representa uma estratégia para a conservação ambiental.

Dos 20 táxons obtidos, oito foram comuns aos períodos de estiagem e de chuvas (Achlya proliferoides, Brevilegnia longicaulis, Dictyuchus pseudodictyon, Plectospira myriandra, Pythiogeton dichotomum, P. ramosum, P. uniforme e $P$. utriforme). Doze foram exclusivos e ocorreram em apenas um dos períodos, seis no período de estiagem (Achlya prolifera, Aphanomyces sp, Brevilegnia linearis, Dictyuchus sp., Globisporangium echinulatume G. perplexum) e seis no período chuvoso (Achlya flagellata, Aphanomyces helicoides, A. keratinophylus, Globisporangium mamillatum, Leptolegniella keratinophila e Saprolegnia luxurians) (Figura 2). 
Tabela 1 - Ocorrência de oomicetos, em cinco coletas, realizadas de agosto de 2014 a junho de 2015, no riacho Mutum, Demerval Lobão, Piauí, Brasil.

\begin{tabular}{|c|c|c|c|c|c|c|c|c|}
\hline \multirow[t]{2}{*}{ Diversidade (D) } & \multicolumn{4}{|c|}{$\begin{array}{c}\text { Coletas no periodo de } \\
\text { estiagem }\end{array}$} & \multicolumn{3}{|c|}{$\begin{array}{l}\text { Coletas no periodo } \\
\text { de chuvas }\end{array}$} & \multirow[b]{2}{*}{ Total Gera. } \\
\hline & 1 & 2 & 3 & Total & 4 & 5 & Total & \\
\hline Achlya flagellata & - & - & - & 0 & 1 & - & 1 & 1 \\
\hline Achlya proliferoides & 4 & 4 & - & 8 & 8 & 4 & 12 & 20 \\
\hline Achlya prolifera & 1 & $\cdot$ & - & 1 & - & - & 0 & 1 \\
\hline Aphanomyces helicoides & $=$ & $\cdot$ & $=$ & 0 & 1 & 1 & 2 & 2 \\
\hline Aphanomyces keratinophylus & - & - & - & 0 & 2 & - & 2 & 2 \\
\hline Aphanomyces sp & - & $\cdot$ & 1 & 1 & - & - & 0 & 1 \\
\hline Brevilegnialinearis & 3 & 4 & - & 7 & - & - & 0 & 7 \\
\hline Brevilegnialongicaulis & 3 & 1 & - & 4 & 2 & - & 2 & 6 \\
\hline Dictyuchus psendodictyon & 1 & - & 1 & 2 & 1 & - & 1 & 3 \\
\hline Dictyuchus sp & $\cdot$ & 1 & - & 1 & - & - & 0 & 1 \\
\hline Globisporangium echimulatum & 1 & - & - & 1 & - & - & 0 & 1 \\
\hline Globisporangium mamillatum & - & - & - & 0 & 1 & 1 & 2 & 2 \\
\hline Globisporangium perplexum & - & - & 1 & 1 & - & - & 0 & 1 \\
\hline Leptolegniella keratinophila & - & - & - & 0 & 2 & - & 2 & 2 \\
\hline Plectospira myriandra & 1 & 1 & - & 2 & 2 & - & 2 & 4 \\
\hline Pythiogeton dichotomum & - & 3 & 2 & 5 & $\cdot$ & 1 & 1 & 6 \\
\hline Pythiogeton ramosum & 2 & 2 & 4 & 8 & 2 & 3 & 5 & 13 \\
\hline Pythiogeton unlforme & $\cdot$ & 2 & 2 & 4 & 2 & 5 & 7 & 11 \\
\hline Pythiogeton utriforme & 1 & $\cdot$ & - & 1 & $\cdot$ & 2 & 2 & 3 \\
\hline Saprolegnia huxwians & - & - & - & 0 & 1 & - & 1 & 1 \\
\hline Abundảncia (A) & 17 & 18 & 11 & 46 & 25 & 17 & 42 & 88 \\
\hline Riqueza (S) & 8 & 8 & 6 & 14 & 12 & 8 & 14 & 20 \\
\hline
\end{tabular}

O índice de similaridade de Sorensen (Is) entre os períodos de chuvas e de estiagem foi de 66,7\% o que sugere uma similaridade acima da mediana dos táxons nos dois períodos e, consequentemente, uma sazonalidade baixa (33,3\%) dos mesmos. Esses resultados condizem com Nascimento (2010), que encontrou alto valor do índice de similaridade de Sorensen entre áreas (70,6\%), demonstrando que o padrão de distribuição das espécies isoladas manteve um nível de uniformidade entre os isolados. 
Figura 2 - Ocorrência de oomicetos nos períodos de estiagem e de chuva, em cinco coletas realizadas de agosto de 2014 a junho de 2015, no riacho Mutum, Demerval Lobão, Piauí, Brasil.

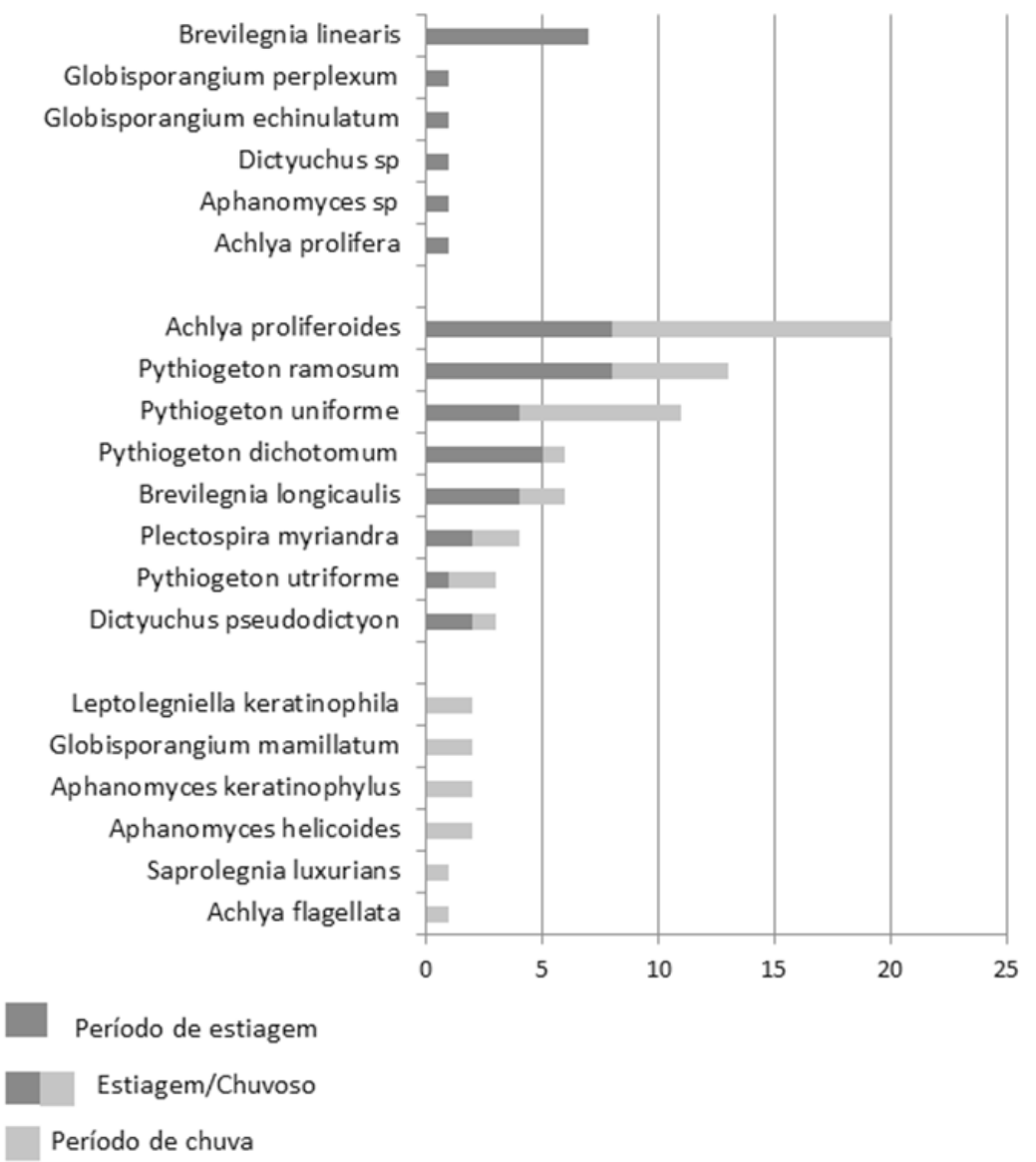

A frequência (\%) e Abundância (A) de oomicetos foram avaliadas utilizando-se a escala de Braun-Blanquet (Tabela 2), que agrupa os táxons em níveis com valores de ocorrência determinados em cinco intervalos. Nesse estudo, apenas uma única espécie foi considerada “ubíqua” (Pythiogeton ramosum) e teve a maior frequência (100\%), duas espécies foram "comuns" (Achlya proliferoides e Pythiogeton uniforme), com frequência de 80\%. Quatro espécies foram "presentes" (Brevilegnia longicaulis, Dictyuchus psendodictyon, Plectospira myriandra e Pythiogeton dichotomum), quatro espécies foram agrupadas em "escassas" (Aphanomyces helicoides, Brevilegnia linearis, Globisporangium mamillatum e Pythiogeto nutriforme), e nove em "raras" (Achlya flagellata, A. prolifera, Aphanomyces keratinophylus, Aphanomyces sp, Dictyuchus sp, Globisporangium echinulatum, G. perplexum, Leptolegniella keratinophila e Saprolegnia luxurians). Nascimento (2010) e Sousa (2015) também relatam Pythiogeton ramosum como o táxon mais frequente (100\%) entre seus achados, de acordo com a escala de Braun-Blanquet.

Por meio do método de iscagem múltipla para o isolamento desses organismos, foi possível observar que todas as espécies obtidas têm hábito sapróbio em substratos celulósicos (semente de sorgo, palha de milho, epiderme de cebola, celofane e papel filtro), queratinosos (cabelo, ecdise de cobra e escama de peixe) e quitinosos (asa de cupim) (Tabela 3). Nesse estudo, quanto ao tipo de substrato, predominaram colonizações nos celulósicos com 48, destacando-se palha de milho com colonizações por 12 táxons (Achlya proliferoides, Brevilegnia linearis, B. longicaulis, Dictyuchus psendodictyon, Globisporangium mamillatum, G. perplexum, Plectospira myriandra, Pythiogeton dichotomum P. ramosum, P. uniforme, P. utriforme e Saprolegnia luxurians) e papel filtro também com doze táxons (Acblya proliferoides, Brevilegnia linearis, B. longicaulis, Dictyuchus pseudodictyon, Globisporangium mamillatum, Leptolegniella keratinophila, Plectospira myriandra, Pythiogeton dichotomum, P. ramosum, P. uniforme, P. utriforme e Saprolegnia luxurians). 
Tabela 2 - Classificação da frequência de oomicetos do riacho Mutum, segundo a Escala de Braun-Blanquet.

\begin{tabular}{|c|c|c|c|}
\hline ESCALA & FREQUÊNCLA $(\%)$ & TÁXONS & TOTAL \\
\hline UBÍQUAS & $80,1-100$ & Pythiogeton ramosum; & 1 \\
\hline COMUNS & $60,1-80$ & $\begin{array}{l}\text { Achlya proliferoides; } \\
\text { Pythiogeton uniforme; }\end{array}$ & 2 \\
\hline PRESENTES & $40,1-60$ & $\begin{array}{l}\text { Brevilegnia longicaulis } \\
\text { Dictyuchus pseudodictyon } \\
\text { Plectospiramyriandra } \\
\text { Pythiogeton dichotomum }\end{array}$ & 4 \\
\hline ESCASSAS & $20,1-40$ & $\begin{array}{l}\text { Aphanomyces helicoides } \\
\text { Brevilegnialinearis } \\
\text { Globisporangium mamillatum } \\
\text { Pythiogeton utriforme; }\end{array}$ & 4 \\
\hline RARAS & $0,1-20$ & $\begin{array}{l}\text { Achlya flagellata } \\
\text { Achlya prolifera } \\
\text { Aphanomyces keratinophylus } \\
\text { Aphanomyces sp } \\
\text { Dictyuchus sp } \\
\text { Globisporangium echinulatum } \\
\text { Globisporangium perplexum } \\
\text { Leptolegniella keratinophila } \\
\text { Saprolegnia luxurians }\end{array}$ & 9 \\
\hline RIQUEZA (S) & & & 20 \\
\hline
\end{tabular}

Semente de sorgo foi colonizada por 11 táxons (Achlya flagellata, A. proliferoides, A. prolifera, Brevilegnia linearis, B. longicaulis, Plectospira myriandra, Pythiogeton dichotomum, P. ramosum, P. uniforme, P. utriforme e Saprolegnia luxurians). Já epiderme de cebola foi colonizada por oito táxons (Achlya flagellata, A. proliferoides, Brevilegnia linearis, B. longicaulis, Dictyuchus sp, Globisporangium echinulatum, Pythiogeton ramosum e P. uniforme). Enquanto que papel celofane teve menor número de táxons colonizado, apenas quatro (Pythiogeton ramosum, P. uniforme, P. utriforme e Saprolegnia luxurians).

Os substratos queratinosos foram colonizados por 14 táxons; fio de cabelo teve registro de apenas um táxon (Achlya proliferoides). Ecdise de cobra teve registro de oito táxons (Achlya proliferoides, Aphanomyces helicoides, A. keratinophylus, Aphanomyces sp, Leptolegniella keratinophila, Pythiogeton ramosum, $P$. uniforme e $P$. utriforme), enquanto que escama de peixe foi colonizada por representantes de três táxons (Leptolegniella keratinophila, Pythiogeton ramosum e P. uniforme).

Os substratos quitinosos tiveram oito colonizações, todas em asa de cupim (Achlya proliferoides, Aphanomyces helicoides, A. keratinophylus, Brevilegnia linearis, Leptolegniella keratinophila, Pythiogeton dichotomume P. ramosume P. uniforme).

Ao representar a diversidade de um determinado local, a utilização de uma única isca, ou substrato, tem restrições porque influencia a presença de organismos zoospóricos, excluindo aqueles que necessitam de outros nutrientes. Espécies adaptadas ou restritas a diferentes fontes de nutrientes podem coexistir e fazer parte da mesma comunidade (Marano et al., 2008). Em uma mesma comunidade podem existir organismos com hábitos sapróbios e colonizar determinados substratos celulósicos, queratinosos ou quitinosos, onde um organismo pode ter mais afinidade por um único tipo de substrato específico. 
Tabela 3 - Diversidade de oomicetos em substratos celulósicos, queratinosos e quitinosos, em cinco coletas bimestrais, de agosto de 2014 a junho de 2015, no riacho Mutum.

\begin{tabular}{|c|c|c|c|}
\hline \multicolumn{2}{|c|}{ SUBSTRATOS } & TAXONS & Total \\
\hline \multirow{5}{*}{$\begin{array}{l}8 \\
8 \\
0 \\
0 \\
0 \\
0\end{array}$} & $\begin{array}{l}\text { Semente de } \\
\text { sorgo }\end{array}$ & $\begin{array}{c}\text { Achlya flagellata } \\
\text { Achlya proliferoides } \\
\text { Achlya prolifera } \\
\text { Brevilegnia lineuris } \\
\text { Bravilegnia longicaulis } \\
\text { Plectospira myriandra } \\
\text { Pythiogeton dichotomum } \\
\text { Pythiogeton ramosum } \\
\text { Pythiogeton unfforme } \\
\text { Pythiogeton utriforme } \\
\text { Saprolegnia luxurians }\end{array}$ & 11 \\
\hline & Palha de milho & $\begin{array}{l}\text { Achlya proliferoides } \\
\text { Brevilegnia linearis } \\
\text { Brevilegnia longicaulis } \\
\text { Dictyuchus pseludodictyon } \\
\text { Globisporangium mamillatum } \\
\text { Globisporangium perplexum } \\
\text { Plectospira myriandra } \\
\text { Pythiogeton dichotomum } \\
\text { Pythiogeton ramosum } \\
\text { Pythiogeton uniforme } \\
\text { Pythogeton utriforme } \\
\text { Saprolegnia luxurans }\end{array}$ & 12 \\
\hline & $\begin{array}{l}\text { Epiderme de } \\
\text { cebola }\end{array}$ & $\begin{array}{c}\text { Achlya flagellata } \\
\text { Achlya proliferoides } \\
\text { Brevilegnia linearis } \\
\text { Brevilegnia longicaulis } \\
\text { Dictyuchus sp. } \\
\text { Globisporangiam echinulatum } \\
\text { Pythiogeton ranosum } \\
\text { Pythiogeton uniforme }\end{array}$ & 8 \\
\hline & Celofane & $\begin{array}{l}\text { Pythiogeton ramosum } \\
\text { Pythiogeton uniforme } \\
\text { Pythiogeton ufriforme } \\
\text { Saprolegnia luxurians }\end{array}$ & 4 \\
\hline & Papel filtro & $\begin{array}{l}\text { Achlya proliferoides } \\
\text { Brevilegnia lnearis } \\
\text { Brevilegnia longicaulis } \\
\text { Dictyuchus pseudodictyon } \\
\text { Globisporangium mamillatum } \\
\text { Leptolegniella keratinophila } \\
\text { Plectospira myriandra; } \\
\text { Pythiogeton dichotomum } \\
\text { Pythiogeton ramoston } \\
\text { Pythiogeton uniforme } \\
\text { Pythiogeton utriforme } \\
\text { Saprolegnia luxurians }\end{array}$ & 12 \\
\hline \multirow{3}{*}{ 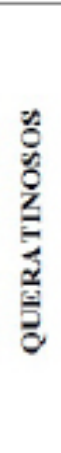 } & Cabelo & Achlya proliferoides & 1 \\
\hline & Ecdise de cobra & $\begin{array}{c}\text { Achlya proliferoides } \\
\text { Aphanomyces helicoides } \\
\text { Aphanomyces keratinophylus } \\
\text { Aphanomyces sp. } \\
\text { Leptolegniella keratinophila } \\
\text { Pythiogeton ramosum } \\
\text { Pythiogeton uniforme } \\
\text { Pythiogeton utriforme }\end{array}$ & 8 \\
\hline & Escama de peixe & $\begin{array}{c}\text { Leptolegniella keratinophila } \\
\text { Pythiogeton ramosun } \\
\text { Pythiogeton uniforme }\end{array}$ & 3 \\
\hline 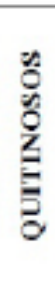 & Asa de cupim & $\begin{array}{c}\text { Achlya proliferoides } \\
\text { Aphanomyces helicoldes } \\
\text { Aphanomyces karatinophylus } \\
\text { Brevilegnia linearis } \\
\text { Leptolegniella keratinophila } \\
\text { Pythiogeton dichotomum } \\
\text { Pythiogeton ramosum } \\
\text { Pythiogeton uniforme }\end{array}$ & 8 \\
\hline
\end{tabular}

GAIA SCIENTIA (2017). VOLUME 11(2): 162-176 
Nos substratos queratinosos, em escama de peixe foram registradas três $(2,70 \%)$ ocorrências, e a menor frequência de colonização ocorreu em cabelo, com duas (1,80\%). Nos substratos quitinosos, em asa de cupim foram registrados oito (7,20\%). Esses dados concordam com as descrições de Rocha (2002), Negreiros (2008), Pereira (2008), Sales (2009) e Trindade Jr. (2013) em estudos com organismos zoospóricos no Piauí, onde relataram frequências similares de colonização.

Tabela 4 - Ocorrência de oomiceto em substratos celulósicos, queratinosos e quitinosos, em cinco coletas bimestrais, de agosto de 2014 a junho de 2015.

\begin{tabular}{|c|c|c|c|c|c|c|c|c|c|}
\hline \multicolumn{2}{|c|}{ SUBSTRATOS } & \multicolumn{5}{|c|}{ Coletas } & \multicolumn{3}{|c|}{ Total $(\%)$} \\
\hline \multirow{6}{*}{ CELULOSICOS } & & 1 & 2 & 3 & 4 & 5 & (111) & $(100)$ & \multirow{6}{*}{80,18} \\
\hline & Semente de sorgo & 8 & 6 & 1 & 9 & 6 & 30 & 27,02 & \\
\hline & Palha de milho & 4 & 4 & 2 & 7 & 7 & 24 & 21,62 & \\
\hline & Epiderme de & 3 & 8 & 0 & 1 & 0 & 12 & 10,81 & \\
\hline & Celofane & 0 & 0 & 1 & 0 & 3 & 4 & 3,60 & \\
\hline & Papel filtro & 3 & 3 & 3 & 8 & 2 & 19 & 17,11 & \\
\hline \multirow{3}{*}{ QUERATINOSOS } & Cabelo & 0 & 0 & 0 & 2 & 0 & 2 & 1,80 & \multirow[t]{3}{*}{12,61} \\
\hline & Ecdise de cobra & 0 & 0 & 1 & 4 & 4 & 9 & 8,10 & \\
\hline & Escama de peixe & 0 & 0 & 0 & 1 & 2 & 3 & 2,70 & \\
\hline QUITINOSOS & Asa de cupim & 0 & 2 & 2 & 2 & 2 & 8 & 7,20 & 7,21 \\
\hline
\end{tabular}

A distribuição das espécies de oomicetos, nos compartimentos água e solo, foi visivelmente destacada com maior ocorrência no solo (Figura 3). Na coleta 1, houve maior ocorrência nas amostras de solo (11) do que nas amostras de água (6). Já na coleta 2, houve um aumento da ocorrência desses organismos no solo (16), enquanto na água houve queda (3). O número de incidência no solo na coleta 3 teve redução (11), porém, curiosamente, nas amostras de água, nessa mesma coleta, não foi obtido nenhum isolamento. Na coleta 4, o número de isolamentos no solo se manteve constante (11) e na água houve aumento (14), também nessa coleta, foi registrado o mais alto valor de ocorrência (25). Já na coleta 5, as amostras de solo aumentaram em número de ocorrências (14), diferentemente das amostras de água que diminuíram consideravelmente (3) em relação à coleta anterior. Esses dados concordam com os achados de Rocha (2002), Pereira (2008) e Sales (2009), os quais relataram maior ocorrência de organismos zoospóricos em compartimentos de solo.

Figura 3 - Frequência de oomicetos em amostras de água e de solo, em cinco coletas, no riacho Mutum, Demerval Lobão, Piauí, Brasil.

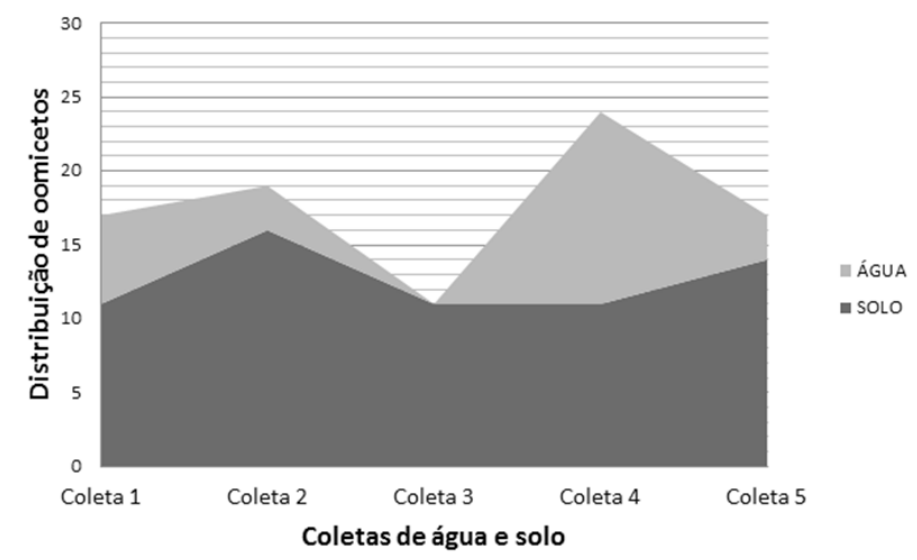


Prabhuji (2011) afirma que os oomicetos têm uma afinidade definitiva por água. Em concordância Dick (1968) tem mostrado de forma conclusiva a afinidade de oomicetos com a água e indicou que o ambiente aquático é necessário para muitas fases do ciclo de vida desses organismos, como a germinação de propágulos, crescimento vegetativo, a esporulação e a disseminação de propágulos.

Provavelmente as amostras de solo contêm uma quantidade de matéria orgânica mais favorável ao encontro de oomicetos, favorecendo o isolamento destes, seria um dos fatores que poderia explicar maior ocorrência nessas amostras. Além disso, a presença de esporos de resistência diminui a influência de fatores limitantes que possam afetar esses organismos. Já em habitats aquáticos, de acordo com Prabhuji (2011), os oomicetos são menos propensos a experimentar condições desfavoráveis, por isso desenvolvem menos quantidade de propágulos de repouso, tais como gemas e oósporos.

Para Reeser et al. (2011), existe uma grande complexidade nos processos que ocorrem em fontes de água, como interações entre espécies, tornando difícil avaliar o efeito de determinado fator abiótico sobre a dinâmica populacional desses oomicetos.

A média das temperaturas do ar registradas nas cinco coletas variou de $33^{\circ} \mathrm{C}$ nas coletas 4 e 5 , a $38^{\circ} \mathrm{C}$, na coleta 2 (Figura 4). Os valores de precipitação foram registrados para as cincos coletas, $1(1 \mathrm{~mm}), 2(33,3 \mathrm{~mm})$, $3(69,2 \mathrm{~mm}), 4(250,7 \mathrm{~mm})$ e $5(115,4 \mathrm{~mm})$. Na coleta 3, a precipitação aumentou, a temperatura diminuiu e a abundância foi a menor registrada. Já na coleta 4, houve correlação positiva com a abundância e a precipitação que aumentaram e negativa com a temperatura, que diminuiu (Figura 4).

Figura 4 - Precipitação, temperatura e abundância registradas em cinco coletas, no período de agosto de 2014 a junho de 2015, no riacho Mutum, Demerval Lobão, Piauí, Brasil.

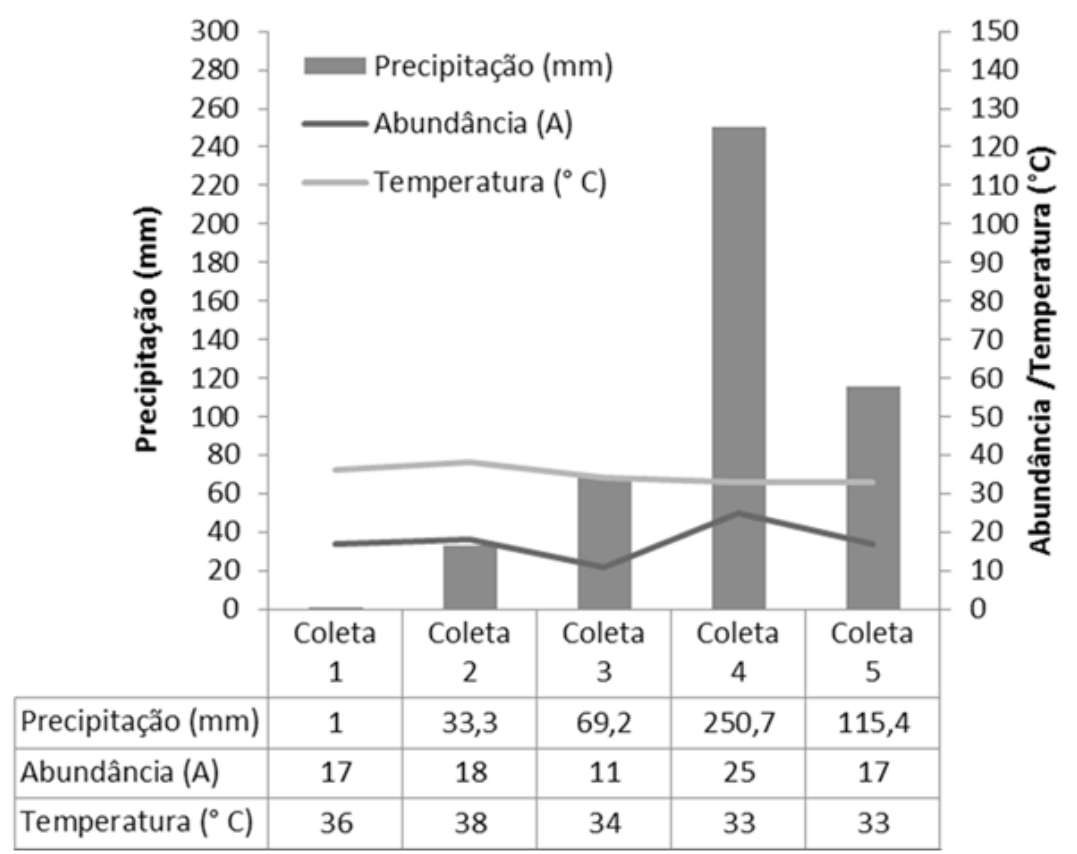

Trindade Jr. (2013) apresenta dados semelhantes em estudos realizados sobre a diversidade de organismos zoospóricos em lagoas de Teresina, em oito coletas, e afirma que não houve diferença significativa entre as temperaturas nas coletas, nas estações chuvosa e seca, embora o índice pluviométrico registrado tenha variado de zero a $247 \mathrm{~mm}$. Existe diversidade de posicionamento sobre a influência dos fatores abióticos (temperatura e precipitação) entre os autores. Para Pires-Zottarelli (1999) o baixo teor de umidade do solo e altas temperaturas, podem afetar a ocorrência desses organismos nos ecossistemas e influenciar em sua diversidade. Durante a 
estação chuvosa, com umidade adequada e baixa temperatura, os propágulos de descanso em solos germinam e produzem zoósporos em abundância (Prabhuji 2011). Ao contrário, Rooney e McKnight (1972), observaram que um aumento na temperatura e a diminuição do oxigênio dissolvido na água, são fatores que aumentam o número de organismos zoospóricos. Porém, diferentemente dos autores mencionados acima, Khallil (1990) afirmou que a temperatura não interferia na ocorrência de organismos zoospóricos na água, considerando outros fatores abióticos como os responsáveis pela distribuição desses organismos.

\section{Conclusão}

Por meio da análise da população dos oomicetos do riacho Mutum, foi possível verificar que fatores ambientais podem influenciar na abundância e distribuição desses organismos. A comunidade de oomicetos mostrou mudanças na ocorrência em relação à sazonalidade, sendo mais abundantes no período de estiagem. Observou-se que todos os táxons tem hábito sapróbio e alguns possuem especificidade por um determinado substrato com maior incidência no solo, fato esse corroborado pela literatura analisada. A diminuição de temperatura e aumento da precipitação contribuíram de forma positiva para a abundância de oomicetos.

Essa pesquisa contribui para evidenciar a importância de parâmetros ambientais como fatores determinantes de características que possibilitam conhecimento mais amplo da dinâmica da população de oomicetos.

\section{Agradecimentos}

À Coordenação de Aperfeiçoamento de Pessoal de Nível Superior - CAPES, pela concessão da bolsa ao primeiro autor, aos moradores do Assentamento Mutum que se disponibilizaram a participar dessa pesquisa, por permitirem o acesso às suas propriedades. Ao laboratório de Fungos Zoospóricos da Universidade Federal do Piauí pela infraestrutura oferecida e aos estagiários que auxiliaram nas atividades.

\section{REFERÊNCIAS}

Beakes GW, Honda D and Thines M. 2014. Systematics of the Straminipila: Labyrinthulomycota, Hyphochytriomycota, and Oomycota. In: The Mycota VIII Part A. McLaughlin DJ, Spatafora JW, (Eds.). Berlin: Springer-Verlag, p. 39-97.

Czeczuga B. 2000. Zoosporic fungi growing on freshwater molluscs.Polish Journal of Environmental Studies, $9(3): 151-156$.

Dick, M. W. 1968. Considerations of the role of water on the taxonomy and ecology of the filamentous biflagellate fungi in littoral zones. Veroff.Des.Inst. Fur Meeresf. Bremerhaven 3: 27-38.

Dick MW. 1976. The ecology of aquatic phycomycetes. In: Gareth Jones EB, ed. Recent advances of aquatic mycology. London, p. 513-542.

Dix NJ and Webster J. 1995. Fungal ecology. Cambridge, UK: Cambridge Univ. Press, 549 p. 
James TY, Letcher PM, Longcore JE, Mozley-Standridge SE, Powell MJ, Griffith GW and Vilgays R. 2006. A molecular phylogeny of the flagellated fungi (Chytridiomycota) and the description of a new phylum (Blastocladiomycota). Mycologia, 98(6): 860-871.

Jesus AL, Marano AV, Schoenlein-Crusius IH and Pires-Zotarelli CLA. 2013. A Diversidade de organismos zoospóricos heterotróficos do Parque Estadual das Fontes do Ipiranga, São Paulo, Brasil: novas citações. Hoehnea, 40(1): 167-180.

Jerônimo G H, Jesus ALD, Marano AV, James TY, Souza JID, Rocha SCO and Pires-Zottarelli CLA. 2015. DiversityofBlastocladiomycotaandChytridiomycotafrom Parque Estadual da Ilha do Cardoso, Cananéia, São Paulo State, Brazil. Hoehnea, 42(1): 135-163.

Johnson Jr. TW, Seymour RL and Padgett DE. 2002. Biology and systematic of Saprolegniaceae.

Khallil AMA. 1990. Mycoflora associated with some freshwater plants collected from Delta region (Egypt). Journal of Basic Microbiology, 30(9): 663-674.

Kershaw KA. 1973. Quantitative and dynamic plant ecology. New York: Elsevier Co. 308 p.

Kirk PM, Cannon PF, Minter DW and Stalpers JA. 2008. Dictionary of fungi. 10 ed., United Kingdom: CABI Publishing.

Kiziewicz B. 2012. Frequency and distribution of zoosporic true fungi and heterotrophic straminipiles from river springs. Polish Journal Environmental Studies, 21(4): 295-303.

Letcher PM and Powell MJ. 2001. Distribution of zoosporic fungi in forest soils of the Blue Ridge and Appalachian Mountains of Virginia. Mycologia, 93(6):1029-1041.

Letcher PM and Powell MJ. 2002. Frequency and distribution patterns of zoosporic fungi from moss-covered and exposed forest soils. Mycologia, 94(5): 761-771.

Marano AV, Barrera MD, Steciow MM, Donadelli JL andSaparrat MC. 2008. Frequency, abundance and distribution of zoosporic organisms from Las Cañas stream (Buenos Aires, Argentina). Mycologia, 100(5): 691-700.

Marano AV, Barrera MD, Steciow MM, Gleason FH, Pires-Zottarelli CL and Donadelli JL. 2011. Diversity of zoosporic true fungi and heterotrophic straminipiles in Las Cañas stream (Buenos Aires, Argentina): assemblages colonizing baits. Fundamental and Applied Limnology/Archivfür Hydrobiologie.178(3): 203-218.

Marano AV, Jesus AL, Pires-Zottarelli CLA, James TY, Gleason FH and de Souza JI. 2014a. Phylogenetic relationships of Pythiales and Peronosporales (Oomycetes, Straminipila) within the "peronosporalean galaxy". In: Gareth Jones EB, Hyde KD, Pang K-L (Eds.). Freshwater fungi and Fungal like Organisms. Germany: Gruyter, p. 177-200.

Milanez AI. 1989. Fungos de águas continentais. In: Fidalgo O andBononi VL (coords.). Técnicas de coleta, preservação e herborização de material botânico. Instituto de Botânica, São Paulo (Série Documentos), p. 
$17-20$.

Müller-Dombois D and Ellemberg H. 1974. Mathematical treatment of vegetation data. In: Aims and methods of vegetation ecology. New York: John Wiley \& Sons, p. 211-302.

Mueller GM, Bills GF, Foster MS. 2004. Biodiversity of fungi: inventory and monitoring methods. Burlington, Massachusetts: ElsevierAcademic Press, 777 p.

Nascimento, CA. 2010. Avaliação da diversidade de organismos zoospóricos da Reserva Biológica e Estação Experimental de Mogi Guaçu, Estado do São Paulo, Brasil. Tese de Doutorado, Instituto de Botânica da Secretaria de Estado do Meio Ambiente. 157 p.

Nascimento CA, Gomes EPC and Pires-Zottarelli CLA. 2011. Occurrence and distribution of zoosporic organisms in water bodies from Brazilian Cerrado. Mycologia103(2): 261-272.

Nascimento CA and Pires-Zottarelli CLA. 2012. Diversidade de fungos zoospóricos da Reserva Biológica de Mogi Guaçu, Estado de São Paulo, Brasil. Rodriguésia63(3): 587-611.

Nascimento CA, Gomes EPC, de Souza JI and Pires-Zottarelli CLA. 2012. Zoosporic true fungi and heterotroficstraminipiles assemblages from soil of Brazilian Cerrado areas. Fungal Ecology5(2): 114-123.

Negreiros NC. 2008. Uso sustentável de culturas agrícolas suscetíveis a oomicetos (Oomycota) fitopatogênicos às margens do rio Parnaíba no município de Floriano, Piauí. Dissertação de Mestrado, Mestrado em Desenvolvimento e Meio Ambiente, Universidade Federal do Piauí. 99 p.

Neuhauser S, Glockling SL, Leaño EM, Lilje O, Marano AV and Gleason FH. 2012. Na introduction to funguslike microorganisms. In: Marine Fungi and Fungal-Like Organisms. Jones, E. B. G. and Pang, K. L. (Eds.). Berlin: De Gruyter, p. 137-151.

Pereira AA.2008. Oomicetos (Oomycota) no campo agrícola de Nazária, Piauí: sustentabilidade na prevenção e controle dos fitopatógenos em agricultura familiar. Dissertação de Mestrado, Mestrado em Desenvolvimento e Meio Ambiente, Universidade Federal do Piauí. 74 p.

Pires-Zottarelli CLA. 1999. Fungos zoospóricos dos vales dos rios Moji e Pilões, região de Cubatão, São Paulo, SP, Brasil. Tese de Doutorado, Instituto de Biociências, Universidade Estadual Paulista. 300 p.

Prabhuji SK. 2011. The interdependence of watermoulds occurring in water and soil habitats affecting their population density, distribution and periodicity.Tropical Ecology, 52(3): 311-324.

Reeser PW, Sutton W, Hansen EM, Remigi P and Adams GC. 2011. Phytophthoraspecies in forest streams in Oregon and Alaska. Mycologia, 103(1): 22-35.

Rocha JRS. 2002. Fungos zoospóricos em área de cerrado no Parque Nacional de Sete Cidades, Piauí, Brasil. Tese de Doutorado, Instituto de Biociências, Universidade de São Paulo. 266 p.

Rocha JRS, Sousa NDC, Negreiros NC, Santos LA, Pereira AA, Sales PCL and Trindade- Júnior OC. 2014. The 
genus Pythiogeton (Pythiogetonaceae) in Brazil.Mycosfera, 5(5): 623-634.

Rooney HM and KH. McKnight. 1972. Aquatic phycomycetes of Lily Lake, Utah. GreatBasinNaturalist, 32: 181-189.

Sales PCL. 2009. Potabilidade da água e presença de oomicetos (Oomycota) em poços freáticos nos povoados Banco de Areia, Bacuri e Roncador no município de Timon, Maranhão. Dissertação de Mestrado, Mestrado em Desenvolvimento e Meio Ambiente, Universidade Federal do Piauí. 98 p.

Shearer CA, Descals E, Kohlmeyer B, Kohlmeyer J, Marvanová L, Padgett D and Voglymayr H. 2007. Fungal biodiversity in aquatic habitats.Biodiversity and Conservation, 16(1): 49-67.

Sparrow Jr. FK.1960. Aquatic Phycomycetes. 2 ed. Ann Arbor: University of Michigan Press, 1187.

Sparrow Jr. FK. 1968. Ecology of freshwater fungi. In: Ainsworth GC, Sussman AS, (Eds.). The Fungi vol. 3. New York: Academic Press, p. 41-93.

Sousa NDC. 2015. A percepção da poluição e o impacto sobre os organismos zoospóricos no rio Poti, Teresina- PI. Dissertação de Mestrado, Mestrado em Desenvolvimento e Meio Ambiente, Universidade Federal do Piauí. 182 p.

Stevens RB. 1974. Mycology guidebook. Seattle: University of Washington press. 703 p.

Trindade Jr. OC. 2013. Riscos socioambientais e diversidade de fungos zoospóricos em lagoas de Teresina, Piauí. Dissertação de mestrado, Mestrado em Desenvolvimento e Meio Ambiente, Universidade Federal do Piauí. 170 p. 